\title{
The Effect of Nanosilica Extracted from Sugarcane Bagasse on Formulation of Flat Sheet Nanofiltration Membrane
}

\author{
Hamizah Mokhtar and Ramlah Mohd Tajuddin
}

\begin{abstract}
Today, nanosilica finds use in a variety of different areas such as biomedical, pharmaceutical, polymer, cosmetic, concrete, environmental and material applications. This work presented a laboratory studies on the preparation and extraction of nanosilica from sugarcane bagasse ash by precipitation method. The morphological analysis of sugarcane bagasse nanosilica (SCBN) was carrying out to be used as the additive in formulation of nanofiltration flat sheet membrane. Flat sheet membrane was fabricated via phase inversion technique. The effect of SCBN on the surface properties were observed and investigated. Membrane cross-section area and SCBN distribution were determined by scanning electron microscope (SEM). The analysis of SEM shows that the addition of different percentage of SCBN apparently changed the surface structure of the membrane particularly at sub layer and top layer. The performance of membranes was measured using flux pure water test and $\mathrm{NaCl}$ rejection test. The results indicate that the addition of $3 \% \mathrm{SCBN}$ give the best rejection of $75.35 \%$ and $42.65 \mathrm{~L} / \mathrm{m}^{2} \mathrm{hr}$ water flux.
\end{abstract}

Index Terms-Additive, flat sheet, membrane, nanofiltration, sugarcane bagasse nanosilica.

\section{INTRODUCTION}

Recently, nanoparticles have attracted a lot of researcher due to the innovative potential uses of particles in nanometer level. Nanosilica has been widely used in polymer, concrete and as inorganic filter to improve the properties of polymeric and mechanical properties. Nanosilica which has a maximum dimension of about $30 \mathrm{~nm}$, is an organic material that has promise of beneficial properties such as excellent stability, high chemical clarity, large surface area, strong adsorption and good dispersal ability [1]. The increasing interest in environmental-friendly materials has motivated in development of nanosilica from various organic materials. Nanosilica can be extracted from rice husk, sorghum vulgarae seed heads and bagasse ash by precipitation method, biodigestion and sol-gel process. The beneficiation of sugarcane bagasse ash (SCBA) has been used in many applications. SCBA is rich in silica approximately $60 \%$ which can be an economically raw material for production of silica gels and powders. Silica can be extracted at low

Manuscript received September 19, 2015; revised December 20, 2015 This work was supported in part by the Ministry of Higher Education.

Hamizah Mokhtar is with the Infrastructure Engineering and Sustainable Management (IIESM), Universiti Teknologi MARA, 40000 Shah Alam, Selangor, Malaysia (e-mail: ami_872001@yahoo.com.my).

Ramlah Mohd Tajuddin was with the Faculty of Civil Engineering, Universiti Teknologi MARA, 40000 Shah Alam, Selangor, Malaysia (e-mail ramlah160@salam.uitm.edu.my). temperature using low energy process due to its amorphous properties [2]. The objectives of this study are to prepare sugarcane bagasse nanosilica (SCBN) using precipitation method and to characterize the SCBN for membrane formulation. For environmental friendly and sustainable materials, membrane can be formulating using organic additive SCBN to replace the synthetic additive commonly used. Commonly used additive in formulation of membrane are silica, cellulose, polymer, carbon nanotube, zeolite, graphite, and metal oxide nanoparticles [1].

Types of solvent and material use in the development of membrane are very significant parameter as to obtain high performance membrane. Membranes are proven to have ability to treat wastewater effectively; however, there is weakness on membrane where its hydrophobic absorption may cause fouling [3]. Some modification of membrane surface is the common methods that been used to minimize the fouling problem hence increase hydrophobic properties of membrane. One of the modification methods is addition or substitution of additive into membrane formulation. The addition of small amount of additive into dope formulation may lead to significant improvement to the performance and characteristics of membrane [1], [4]. Furthermore, the addition of additive can change the phase separation kinetic, thermodynamic mechanism and solvent capacity and also enhance pore distribution, mechanical and physical properties [1], [5]. Previous studies reported that the addition of silica in membrane formulation will improve anti-fouling mechanism, permeability, surface porosity, molecular weight cut off and resistance ability [3]. However, different concentration of silica produces different effect on membrane properties. Therefore, in this study SCBN were used as additive in nanofiltration membrane. Then, the effect of SCBN to membrane morphology and performance were investigated.

\section{RESEARCH METHODS}

\section{A. Synthesis Pure Silica from Sugarcane}

Sugarcane bagasse samples were stirred in sodium hydroxide solution and boiled for $3 \mathrm{~h}$ [2]. The solution was filtered and the residue was washed with boiling water. The filtrate was allowed to cool down to room temperature and $\mathrm{H} 2 \mathrm{SO} 4$ was added until $\mathrm{pH} 2$ and then added $\mathrm{NH} 4 \mathrm{OH}$ until $\mathrm{pH} 8.5$ [2]. Then, the filtrate was dried at $120^{\circ} \mathrm{C}$ for $12 \mathrm{~h}$.

\section{B. Preparation of Nanosilica}

Nanosilica was prepared by refluxing with $\mathrm{HCl}$ for $4 \mathrm{~h}$ and then washed frequently using deionised water to make it acid 
free. After that the nanosilica sample was dissolved in $\mathrm{NaOH}$ by stirring continuously for $10 \mathrm{~h}$ on a magnetic stirrer [2]. Then concentrated $\mathrm{H}_{2} \mathrm{SO}_{4}$ was added to adjust $\mathrm{pH}$ in the range of 7.5-8.5. The precipitated nanosilica was washed repeatedly with warm deionised water until the filtrate became completely alkali free. The deionised water was used time after time for washing process. The nanosilica was dried at 50 ${ }^{\circ} \mathrm{C}$ for $48 \mathrm{~h}$ in the oven [2].

\section{Morphology of Nanosilica}

Field Emission Scanning Electron Microscopy (FESEM) and energy-dispersion x-ray analysis (EDX) was used to analyse the morphology and material characterization of synthesized SCBN. The SCBN sample was scanned along the path of the electron beam and photograph of the sample was taken.

\section{Formulation of Nanofiltration Membrane}

In this study, the formulation of nanofiltration membrane consist of polysulfone (Psf), N,N-Dimethylacetamide (DMAc), poly-vinylpyrrolidone (PVP) and Sugarcane bagasse nanosilica (SCBN). The Psf was dried by temperature $50^{\circ} \mathrm{C}$ for $24 \mathrm{~h}$ before use in casting formulation. Then, casting formulations were prepared by dissolving the ratio between Psf: PVP: DMAc: SCBN. Table I shows the dope composition formulae for membrane formulation. Psf was added into the vessel containing the solvent DMAc and stirred continuously by using a magnetic stirrer to make sure the Psf is well mixed. This procedure was repeated until all the required amount of polymer is added. This process was controlled at desired range $60^{\circ} \mathrm{C}$ temperature and $400 \mathrm{rpm}$ speed. The solution was stirred until it become homogeneous. Next, the additive, PVP was added in the solution and followed by additive SCBN. Then, the mixing process was continued until a homogeneous solution is obtained. After that the dope solution was poured and cooled in a clean one liter storage glass bottle. The above steps is surface treatment of membrane fabrication process which involved evaporating the solvent on the membrane surface by allowing air being sucking out of the air hood. Later on, the dope solution undergo coagulation steps where the membrane sheets were immersed into appropriate liquid at appropriate temperature so that the membrane will harden and detach from the casting plate. The dope solution was cast using casting knife with range thickness $100-125 \mu \mathrm{m}$ and immersed into coagulation bath containing distilled water under room temperature. The flat sheet membrane was air-dried for 24 hour at room temperature $\left(27^{\circ} \mathrm{C}\right)$. Finally, the membranes then were cut in ring shape about $6 \mathrm{~cm}$ diameters before placed in the cell for pure water flux and $\mathrm{NaCl}$ rejection.

\section{E. Performance and Morphology of Nanofiltration Membrane}

The performance of flat sheet nanofiltration membrane with a variation of dope composition has been characterized by using pure water flux test and Salt $(\mathrm{NaCl})$ Rejection Test. Pure water flux calculation is $F=Q /(A \times \Delta t)$, where $\mathrm{F}$ is permeation flux for distilled water and wastewater $\left(\mathrm{L} / \mathrm{m}^{2} \mathrm{hr}\right)$, $Q$ is volumetric flow rate of permeation solution, $A$ is crossflow surface area $\left(\mathrm{m}^{2}\right)$ and $\Delta t$ is time interval on which permeate volume is taken (hr) [6]. Whereas, the $\mathrm{NaCl}$ rejection can be defined as percentage of solute concentration reduction of permeate stream relative to feed stream and can be calculated as $\mathrm{SR}=1-(C P / C F) \times 100 \%$, where $C P$ and $C F$ are solute concentration in the feed and permeate solution respectively [7]. Morphology of nanofiltration membrane was examined by Scanning Electron Microscopy (SEM). The nanofiltration membrane were fractured and immersed in liquid nitrogen carefully. Then, the sample was coated by sputtering gold to prevent charging of the surface by the electron beam. The nanofiltration images were captured in term of surface morphology and cross-sections morphology [8].

TABLE I: DOPE COMPOSITION

\begin{tabular}{ccccc}
\hline \hline DOPE & DMAC $(\%)$ & PSF $(\%)$ & PVP $(\%)$ & SCBN $(\%)$ \\
\hline S1 & 21 & 71 & 7 & 1 \\
S2 & 21 & 71 & 6 & 2 \\
S3 & 21 & 71 & 5 & 3 \\
S4 & 21 & 71 & 4 & 4 \\
S5 & 21 & 71 & 3 & 5 \\
S6 & 21 & 71 & 2 & 6 \\
\hline \hline
\end{tabular}

\section{RESULTS AND DISCUSSIONS}

\section{A. Morphology of Sugarcane Bagasse Nanosilica}

The image of SCBN analyse using FESEM was shown in Fig. 1. The surface of SCBN showed the rough surface which benefit to the high surface area resulted easy to react with solution. EDX profile of SCBN contained the elements of $\mathrm{Si}$, $\mathrm{O}, \mathrm{C}$ and $\mathrm{Mg}, \mathrm{K}$ and $\mathrm{Au}$ as shown in Fig. 2. Both $\mathrm{Si}$ and $\mathrm{O}$ peaks correspond to the silica which $20.28 \%$. The dominant signals originate from gold $\mathrm{Au}(\mathrm{Au})(65.0 \%)$ is due to gold coating. Table II shows the weight percentage of each element in SCBN.

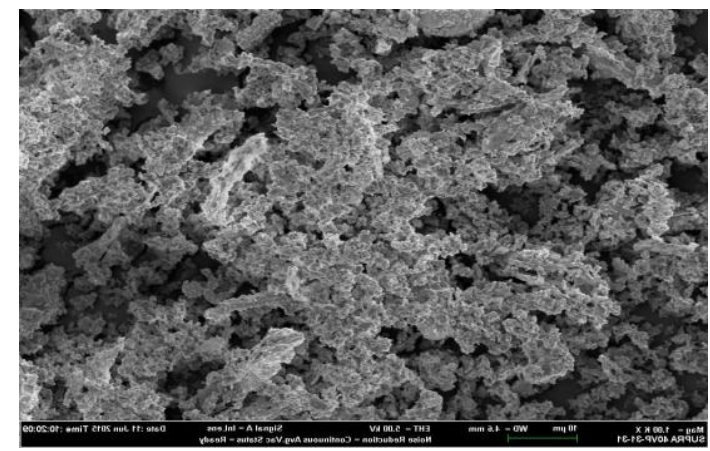

Fig. 1. FESEM image of sugarcane bagasse nanosilica (SCBN)

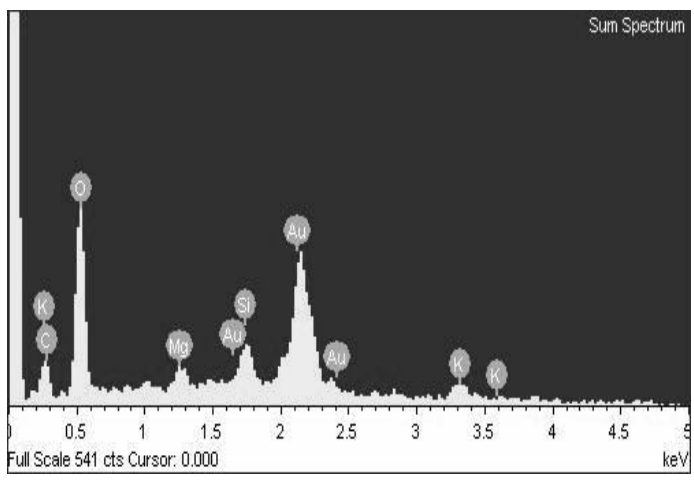

Fig. 2. EDX of sugarcane bagasse nanosilica (SCBN). 
TABLE II: EDX PROFILE OF SCBN

\begin{tabular}{ccc}
\hline \hline ELEMENT & WEIGHT $(\%)$ & ATOMIC $(\%)$ \\
\hline C K & 1.48 & 6.29 \\
O K & 15.80 & 50.31 \\
MG K & 1.53 & 3.20 \\
SI K & 4.48 & 8.12 \\
K K & 11.71 & 15.26 \\
AU M & 65.00 & 16.81 \\
TOTAL & 100.00 & \\
\hline \hline
\end{tabular}

\section{B. Performance and Morphology Studies of Nanofiltration} Membrane

The effect of SCBN as an additive on the pure water flux (PWF) and salt rejection performance is illustrated in Fig. 3. From the figure, the flux is increase when the SCBN percentage increases up to $3 \%$. The PWF of $1 \% \mathrm{SCBN}$ is $28.90 \% \mathrm{~L} / \mathrm{m}^{2} \mathrm{hr}, 2 \% \mathrm{SCBN}$ is $33.62 \mathrm{~L} / \mathrm{m}^{2} \mathrm{hr}$ and $3 \% \mathrm{SCBN}$ is $42.65 \mathrm{~L} / \mathrm{m}^{2} \mathrm{hr}$, this increases trend might due to the SCBN $3 \%$ is more hydrophilicity compare to the other percentage of SCBN. After addition of $4 \%$ to $6 \% \mathrm{SCBN}$, the PWF were slightly similar with average flux of $42.54 \mathrm{~L} / \mathrm{m}^{2} \mathrm{hr}$. Previous study reported by [9] shows that $2 \%$ of silica give the best pure water flux of $25.30 \mathrm{~L} / \mathrm{m}^{2} \mathrm{hr}$. This is due to the interaction between contaminants and membrane surface were reduced by silica particles of the membrane surface [9]. In this study, the optimum flux rate can be considered at membrane which contains $3 \%$ of SCBN which indicate that hydrophilicity of the membrane increases and consequently it attract water molecules into the composite membrane. Moreover hydrophilicity properties also may facilitate water penetration through the membrane thus enhancing the flux.

Fig. 3 also describes the effect on addition of SCBN in terms of percentage of salt rejection. The results indicated that at the first $3 \%$ of $\mathrm{SCBN}$, the salt rejection were slightly similar approximately $75.33 \%$ rejection. Then, the salt rejection shows gradually decreased from $75.35 \%$ to $71.18 \%$ with addition of $4 \%$ to $6 \% \mathrm{SCBN}$. According to the results, the $3 \% \mathrm{SCBN}$ show the highest $\mathrm{NaCl}$ rejection of $75.35 \%$ due to the optimal mixture of SCBN that result in good hydrophilic properties. The high hydrophilicity membrane may reduce interaction between hydrophobic contaminants and the membrane surface; hence, the rejection properties were improved effectively [9]. This is clearly show by the rejection properties when SCBN is added as additive in nanofiltration membrane. However, with more addition of SCBN result to low $\mathrm{NaCl}$ rejection. This may be due to crystalline effect of the SCBN that has low compatibility when mixed with polymer. Therefore, the small particles can be a small defect and this surely will affect the rejection value.

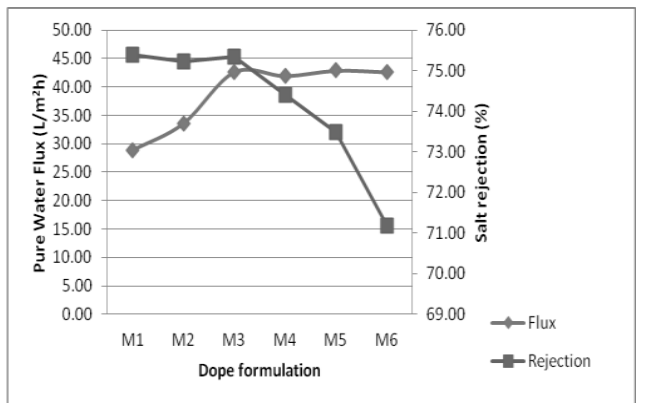

Fig. 3. Pure water permeation and salt rejection of different dope formulation.
The best mixture of nanofiltration membrane that gives the most excellent pure water flux and the highest $\mathrm{NaCl}$ rejection is dope S3. Fig. 4 shows the SEM image of nanofiltration membrane for dope S3 in terms of surface morphology. The SEM image indicates that the membrane have macrovoids and forming a spongy microstructures. The pore sizes are well distributed with the size range between $468 \mathrm{~nm}$ and $919 \mathrm{~nm}$. Fig. 5 shows the SEM image of cross section for dope S3that describes an asymmetric structure consisting of dense top layer, a porous sub-layer and a small portion of spongy -like structure between bottom surface layer. The top layer acts as a separation layer whereas the sub-layer provides the mechanical strength for membrane. The finger-like structure beneath the top surface layer becomes large void near the bottom surface layer. The width of the nanofiltration membrane for dope $\mathrm{S} 3$ is $101 \mu \mathrm{m}$. The result was similar to the previous work by $[9,10]$ showed the membranes consist of a dense skin layer at top surface and short finger-like structure. This relates to high rejection due to the effective rejection that flow through dense skin layer and finger-like structure explaining the high water flux obtained. Previous study by [11] reported that the straight and longest finger like structure give the best permeation compared to short finger like structure. In fact, the smallest pore size at the top layer of membrane lead to the better rejection.

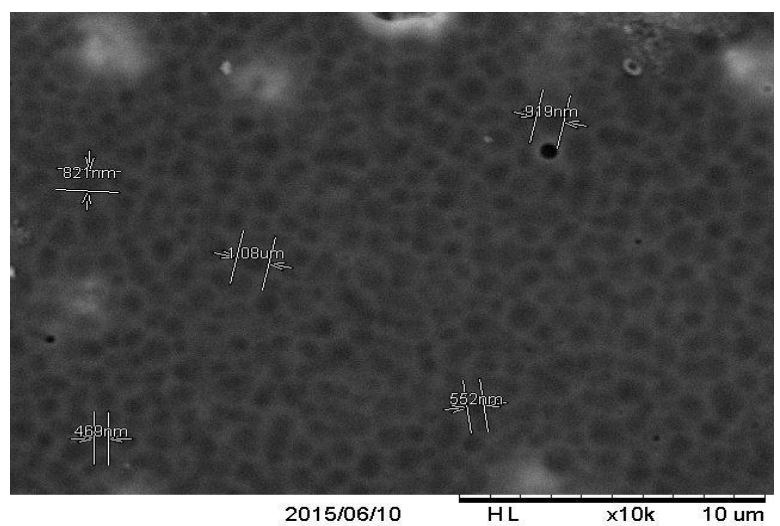

Fig. 4. SEM surface image of dope S3 nanofiltration membrane.

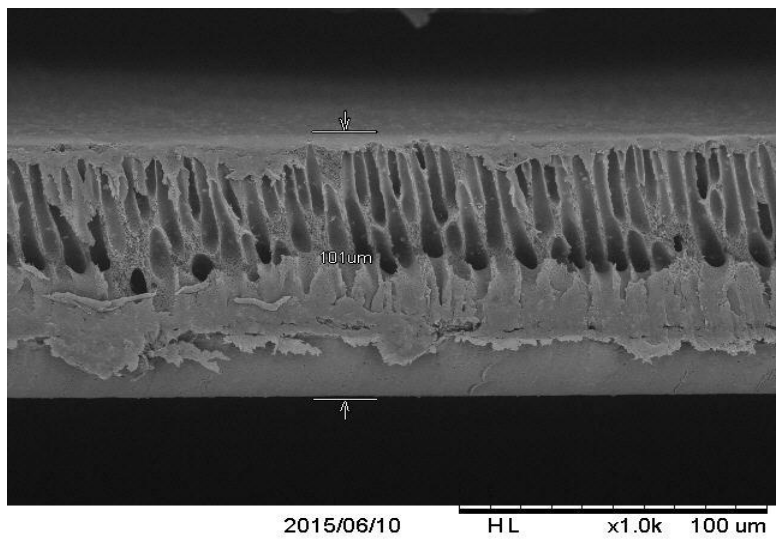

Fig. 5. SEM cross section image of dope S3 nanofiltration membrane.

\section{CONCLUSIONS}

Sugarcane bagasse nanosilica (SCBN) membrane were successfully extracted and prepared using precipitation method. SCBN which is environmental-friendly materials was used as one of additive in membrane formulation. The 
best formulation of nanofiltration flat sheet membrane is dope S3 with composition of $21 \%$ PSf: $71 \%$ DMAc: $5 \%$ PVP: $3 \%$ SCBN. The addition of sugarcane bagasse nanosilica was affecting the performance of the membranes in terms of pure water flux and rejection. The results indicate that the addition of $3 \%$ SCBN give the best rejection of $75.35 \%$ and 42.65 $\mathrm{L} / \mathrm{m} 2 \mathrm{hr}$ water flux. The morphology studies indicates that the addition of nanosilica has enlarged the pore size in sub layer creating finger-like macrovoids which then results in longer sub layer. In this study, the efficient membrane has been produced and can be further used in wastewater treatment process.

\section{ACKNOWLEDGMENT}

The author is grateful to the Institute for Infrastructure Engineering and Sustainable Management (IIESM) and Ministry of Higher Education for funding this research.

\section{REFERENCES}

[1] Z. Harun, M. R. Jamalludin, M. Z. Yunos, M. F. Shohur, and A. F. Ismail, "The effect of amorphous rice husk silica to the polysulfone membrane speration process," Applied Mechanics and Materials, vol. 701, pp. 319-322, 2013.

[2] N. Thuadaij and A. Nuntiya, "Preparation of nanosilica powder from rice husk ash by precipitation method," Chiang Mai J. Sci., vol. 35, no. 1, pp. 206-211, 2008.

[3] A. N. I. Idris, C. M. Kee, and I. Ahmed, "Effect of monosodium glutamate additive on performance of dialysis membrane," Journal of Engineering Science and Technology, vol. 3, no. 2, pp. 172-179, 2008.

[4] K. Kogutid and K. Kuns, "RO and NF membrane fouling and cleaning and pore size distributuion variations," Desalination, vol. 150, pp. 13-120, 2002.

[5] J. Huang, J. Xue, K. Xiang, X. Zhang, C. Cheng, S. Sun, and C. Zhao, "Surface modification of polyethersulfone membrnaes by blending triblock copolymer of methoxyl poly(ethylene glycol)- polyurethanemethoxyl poly(ethylene glycol)," Colloids and Surfaces B: Biointerfaces, vol. 88, no. 1, pp. 315-324, 2011.

[6] H. Yua, Y. Caoa, G. Kanga, J. Liua, M. Li, and Q. Yuana, "Enhancing antifouling property of polysulfone untrafiltration membrane by grafting zwitterionic copolymer via UV-initiated polymerization," Journal of Membrane Sciences, vol. 342, pp. 6-13, 2009.

[7] D. Hellman, "A novel process of membrane fabrication: thermally assisted evaporative phase separation (TAEPS)," Journal of Membrane Science, vol. 230, no. 1-2, pp. 99-101, 2004.

[8] A. I. Ahmad, W. A. N. A. Harris, and O. O. I. B. Seng, "Removal of Dye wastewater of textiles industry using membrane technology," Jurnal Teknologi, UTM 36, pp. 31-44, 2007.

[9] E. Yuliwati, A. F. Ismail, W. J. Lau, B. C. Ng, A. Mataram, and M. A. Kassim, "Effects of process conditions in submerged ultrafiltration for refinery wastewater treatment: Optimization of operating process by response surface methodology," Desalination, vol. 287, pp. 350-361, 2012.

[10] H. Mokhtar, M. N. Abdullah, and R. M. Tajuddin. "Fabrication of flat sheet blend ultrafiltration membrane using DMAc as solvent for aluminium removal," presented at the $4^{\text {th }}$ International Conference on Environmental Research and Technology, Penang, Malaysia, May 27-29, 2015

[11] H. Mokhtar and R. M. Tajuddin, "Flat sheet membrane using silica from sugarcane bagasse as additive: Effect of morphology," International Journal of Scientific and Research Publications, vol. 5 , no. 9, pp. 1-4, September 2015.

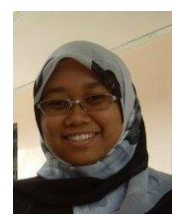

H. Mokhtar obtained her BSc degree in environmental technology from Universiti Teknologi MARA, Shah Alam, Malaysia in 2009. Then received her master of science at Universiti Sains Malaysia, Penang on year 2012. During her master study, she was be given a fellowship from Institute of Postgraduate Studies, Universiti Sains Malaysia.

Currently, she is a $\mathrm{PhD}$ research student at the Faculty of Civil Engineering, Universiti Teknologi MARA, Shah Alam. She also holds a scholarship from the Ministry of Higher Education of Malaysia.

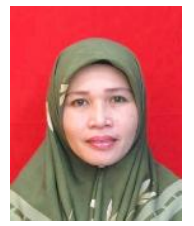

R. M. Tajuddin obtained her master degree in civil engineering from the University of Leeds in 1994 and her PhD in Civil Engineering, University Teknologi Malaysia in 2006

Her areas of research include development of newly membrane, wastewater treatment, tropical public health and phytoremediation techniques.

Associate Professor Tajuddin is currently the coordinator for Postgraduate Programme (research) of the Water Resources Environmental System Division, Faculty of Civil Engineering, Universiti Teknologi MARA. 